\title{
Modeling amplified arbitrary filtered Microwave Photonic links and systems
}

\author{
Erica Sánchez, ${ }^{1 *}$ Daniel Pérez-López, ${ }^{1,2}$ Prometheus \\ DASMAHAPATRA $^{1,2}$ AND JOSÉ CAPMANY ${ }^{1,2}$ \\ ${ }^{1}$ Photonics Research Labs, iTEAM Research Institute, Universitat Politècnica de València, Spain. \\ 2 iPronics Programmable Photonics S. L, Valencia, Spain \\ *ersango@iteam.upv.es
}

\begin{abstract}
Microwave photonics (MWP) links and systems will have more losses as their complexities increase and there will be a need for incorporating optical amplification. Here, we report results of an analytical model developed for amplified arbitrary filtered MWP systems that provides the expressions of the main figures of merit for intensity modulation direct detection. It contemplates the cases of power, intermediate and pre amplification. The model is applied to a long MWP link and then it is evaluated in a MWP reconfigurable filter implemented by means of a programmable waveguide mesh photonic integrated circuit.
\end{abstract}

\section{Introduction}

For several decades Microwave Photonic systems have been described by relatively simple configurations that included a direct or external modulated optical transmitter, a dispersive optical fiber link and a broadband linear optical receiver [1-3]. Their main role was to provide broadband and highly linear end-to-end links, capable of transmitting radiofrequency analog signals leveraging on the unique properties of optical fibers in terms of low loss, electromagnetic immunity and broadband operation [4]. These configurations were well suited for the niche applications in security and defense targeted by early adopters.

However, the surge of new and emerging applications such as 5/6 G mobile communications, Internet of Things (IoT) and industry 4.0 calls for MWP systems with increased complexity [5] that can guarantee a smooth interface between optical fiber based transport networks and wireless edge segments.

Adding complexity in MWP systems implies empowering their photonic cores with the capability of performing in-flight arbitrary optical signal processing tasks, such as filtering, beam-steering, long distance transmission, signal generation/conditioning and ADC to cite some examples. This can be achieved by either fiber-based configurations or, most likely with Photonic Integrated Circuits (PICs). The addition of this processing capability can be modelled by including an arbitrary passive filter transfer function $\mathrm{H}(\omega)$ in the optical part of the link and several works [6-9] have reported the derivation of the fundamental Figures of Merit (FOMs) of these complex systems.

As complexity grows and/or more distance is covered by MWP, losses increase and these systems calls for the incorporation of optical amplification to improve the link performance [7]: Erbium Doped Fiber Amplifier (EDFA) based in fiber links and Semiconductor Optical Amplifiers (SOA) based in systems including PICs. The inclusion of optical amplifiers (OAs) has been considered [10] and experimentally studied [8] in simple MWP links. Moreover, although several works have attempted to model theoretically the key performance metrics to analyze MWP filter links and systems [9], expressions that define in a closed way the effects of these amplifiers to the FOM of the links have not been reached. There is, to our knowledge, no work reported so far on the modelling of amplified arbitrary filtered MWP links. 
In this work, we report the results of an analytical model for amplified complex MWP systems. It provides the expressions for the main FOMs (i.e. RF Gain, Noise Figure (NF) and dynamic range) for the case of intensity modulation and contemplates the cases of power amplification (i.e. OA placed after the transmitter), intermediate amplification (i.e. inside the optical processing/distributing core), and pre amplification (i.e. OA placed before the detector).

The model enables a flexible evaluation of the position on the OA and the overall impact of the performance of each subsystem. As an application example, the model is applied to two specific cases. The first one corresponds to a long MWP link, while the second describes a MWP reconfigurable filter implemented by means of a programmable waveguide mesh PIC [11].

\section{Analytical model}

\subsection{Introductory considerations}

A SOA is an optoelectronic device that can amplify an input light signal. The active region in the device is able to amplify the signal due to the gain generated by stimulated emission. However, the noise generated internally by spontaneous emission, which is also amplified in the device, is added to the output signal. This Amplified Spontaneous Emission (ASE) noise is produced by the amplification process itself and it cannot be avoided [12]. The effect of this noise on the MWP link/system performance is the main focus of this work.

Conventional FOMs for MWP links (gain, noise and dynamic range) are evaluated using general expression of the RF Gain, Noise Figure (NF) (or relative intensity noise, RIN) and Spurious Free Dynamic Range (SFDR), respectively. These FOMs are usually employed to analyze signal degradations due to noise sources [6-10]. For that, we define the RF Gain as the ratio between the input RF power $\left(\mathrm{P}_{\mathrm{RFin}}(\Omega)\right)$ and the output RF photodetected power $\left(\mathrm{P}_{\mathrm{RFout}}(\Omega)\right)$ :

$$
\begin{aligned}
& G_{R F}(\Omega)= \frac{P_{R F o u t}(\Omega)}{P_{R F i n}(\Omega)}=G^{2} G_{R F-P F}^{I} \\
& G_{R F-P F}^{I}(\Omega)=\frac{1}{16}\left(\frac{\eta e P_{i n} \alpha_{m z m} \pi}{\hbar \omega_{o} V_{\pi}}\right)^{2} \sin ^{2}\left(\phi_{d c}\right) . \\
& \\
& \quad R_{\text {in }} R_{\text {out }}\left|\frac{H_{0}\left(\omega_{o}\right) H_{0}^{*}\left(\omega_{o}-\Omega\right)+H_{0}^{*}\left(\omega_{o}\right) H_{0}\left(\omega_{o}+\Omega\right)}{2}\right|^{2}
\end{aligned}
$$

where $\mathrm{G}$ is the gain of the optical amplifier used and $\mathrm{H}_{0}(\omega)$ is the arbitrary transfer function of the optical system in the MWP link $[4,6,9,10]$ that incorporates all the losses (i.e it is given by $H_{0}(\omega)=L^{1 / 2} H(\omega)$, where $H(\omega)$ is a lossless transfer function and L represents the loss coefficient).

On the other hand, the relative intensity noise $\left(\mathrm{RIN}_{\mathrm{TOT}}\right)$ is defined in Eq. (2) and the total output spectral density noise $\left(\mathrm{N}_{\mathrm{TOT}}\right)$ is shown in Eq. (3).

$$
\operatorname{RIN}_{T O T}=\frac{N_{T O T}(f)}{i_{d c}^{2} R_{O U T}}
$$

where $i_{d c}$ is the DC component from the signal current and $R_{\text {OUT }}$ is the load resistance. 


$$
N_{\text {TOT }}=N_{\text {sig-sp }}(f)+N_{s p-s p}(f)+N_{s p-s h o t}(f)+N_{\text {thermal }, i}(f)+N_{\text {thermal }, o}(f)+N_{\text {sig-shot }}(f)
$$

Thermal input $\left(\mathrm{N}_{\text {thermal, } \mathrm{i}}\right)$ and thermal output $\left(\mathrm{N}_{\text {thermal,o }}\right)$ noises generated due to the modulator and the receiver electronics respectively, and signal-shot noise $\left(\mathrm{N}_{\text {s-shot }}\right)$ generated during the photodetection, are common noise sources in any MWP link. Moreover, the spontaneous emission from the optical amplifier generates three types of additional noises in the electrical domain [11]. First, the spontaneous emission beats with the signal during photodetection and produces a noise $\left(\mathrm{N}_{\mathrm{sig} \text {-sp }}\right)$. Secondly, the spontaneous emission noise is beaten with itself $\left(\mathrm{N}_{\mathrm{sp}-}\right.$ sp) during the photodetection stage. Finally, shot noise is produced proportional to the average spontaneous emission noise power $\left(\mathrm{N}_{\mathrm{sp} \text {-shot }}\right)$.

Therefore, final noise figure of the MWP link is evaluated using the above noise expressions and using the general expression of this figure derived in Eq. (4).

$$
N F=\frac{N_{T O T}}{G_{R F} k_{B} T}
$$

Using Eq. (2), the equation of noise figure can be rewritten as a function of the relative intensity noise as:

$$
N F=\frac{\operatorname{RIN}_{T O T} i_{d c}^{2} R_{o u t}}{G_{R F} k_{B} T}
$$

Finally, the link dynamic range can be evaluated with the second and third order SFDRs, using the second-order and third-order output intercept points respectively $\left(\mathrm{OIP}_{2}\right.$ and $\left.\mathrm{OIP}_{3}\right)$ is obtained using Eq. (6) and Eq. (7) [10]:

$$
\begin{aligned}
& S_{F D R_{2}}=\left[\frac{O I P_{2}}{N_{T O T}}\right]^{1 / 2}=\left[\frac{O I P_{2}}{R I N_{T O T} i_{d c}^{2} R_{\text {OUT }}}\right]^{1 / 2} \\
& \text { SFDR }_{3}=\left[\frac{O I P_{3}}{N_{\text {TOT }}}\right]^{2 / 3}=\left[\frac{O I P_{3}}{\operatorname{RIN}_{\text {TOT }} i_{d c}^{2} R_{\text {OUT }}}\right]^{2 / 3}
\end{aligned}
$$

where, $\mathrm{N}_{\text {TOT }}$ is the total noise power density distribution defined in Eq. (3) and the output intercept point (OIP) is the output power at the intersection of the extrapolated small-signal responses of the fundamental tone and the nth-order distortion. The OIPs are obtained using RF photodetected power relative to the signal contribution and relative to the intermodulation distortions ( $\mathrm{IMD}_{2}$ and $\mathrm{IMD}_{3}$ ) expressions [4]. Moreover, the OIPs do not change with respect to the passive case as the output RF powers of both the fundamental and intermodulation terms scale equally with $\mathrm{G}^{2}$.

While in this paper, the FOMs of an amplified filtered MWP link are reported for the case of intensity modulation and direct detection, the same methodology can be applied to extend the model to alternative modulation and detection techniques. The aim of this model is to provide the FOMs of an arbitrary filtered MWP link system containing an optical amplifier such a SOA/EDFA at different positions of a general MWP link as shown in Fig. 1. Point A corresponds to the power-amplification case, point $\mathrm{B}$ is in-line-amplification and point $\mathrm{C}$ represents the pre-amplification case. 


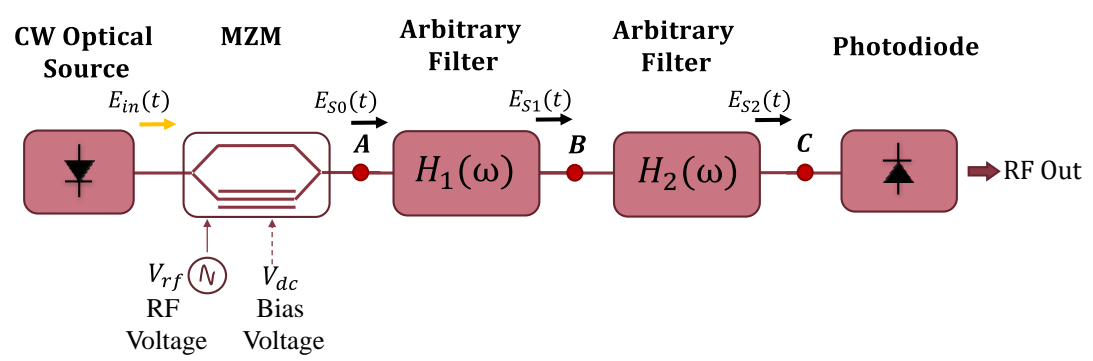

Fig. 1. Schematic of an amplifier filtered MWP system (point A power-amplified, point B in-line amplified and point C pre-amplified) link.

Referring to Fig. 1, the input optical field to the link is defined as:

$$
E_{i n}(t)=\sqrt{P_{i n}} e^{\left(j \omega_{0} t\right)}
$$

where the optical field $E_{\text {in }}(t)$ is the one generated by the optical source and $P_{\text {in }}$ is optical power at frequency $\omega_{0}[10]$.

Then, the Mach-Zehnder Modulator (MZM) modulates the RF information onto the laser intensity [10]. Hence, $\mathrm{E}_{\mathrm{SO}}(\mathrm{t})$ is the optical field at the output of the modulator and it is expressed as:

$$
E_{S 0}(t)=j \sqrt{\alpha_{m z m}} E_{i n}(t) \sin \left(\frac{\phi_{d c}}{2}+\frac{\phi_{r f}}{2} \sin (\Omega t)\right)
$$

with,

$$
B_{n}=(-1)^{n} j^{|n|+1} \sin \left(\frac{\phi_{d c}}{2}+|n| \frac{\pi}{2}\right)
$$

where $\alpha_{\mathrm{mzm}}$ are the losses due to the modulator, $\phi_{\mathrm{dc}}=\left(\pi \mathrm{V}_{\mathrm{dc}}\right) / \mathrm{V}_{\pi}$ and $\phi_{\mathrm{rf}}=\left(\pi \mathrm{V}_{\mathrm{rf}}\right) / \mathrm{V}_{\pi}$ for the bias voltage $V_{\mathrm{dc}}$ and the RF voltage $\mathrm{V}_{\mathrm{rf}}$ respectively, $\mathrm{V}_{\pi}$ is the modulator half-way voltage and $\Omega$ is the angular frequency of the RF tone. Afterwards, the field can be simplified as:

$$
E_{S 0}(t)=j \sqrt{\alpha_{m z m} P_{i n}} e^{j \omega_{0} t} \sum_{n=-\infty}^{\infty} B_{n} J_{n}\left(\frac{\phi_{r f}}{2}\right) e^{j n \Omega t}
$$

\subsection{Power-amplified link/system}

In this case, the amplifier is located in point A, previous to the arbitrary filter/optical core (Fig. 2). 


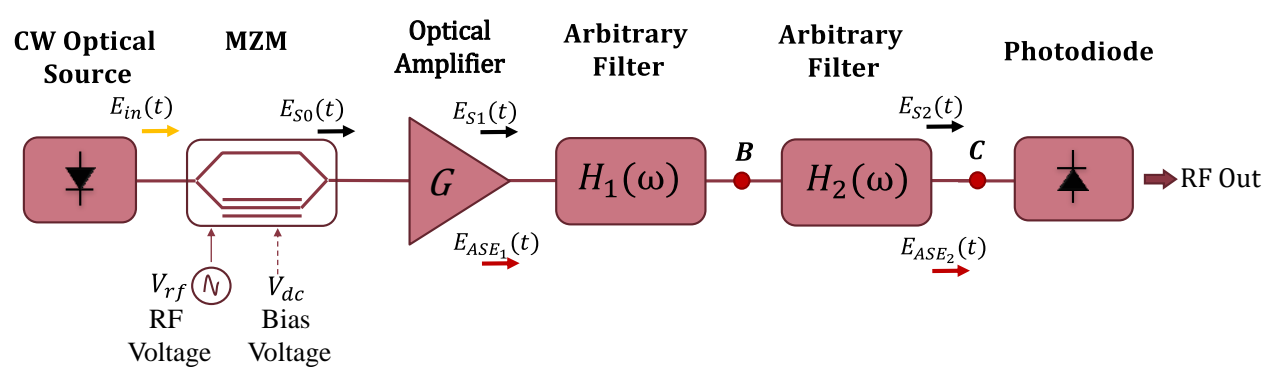

Fig. 2. Schematic of power-amplified filtered MWP link/system for the analysis.

Here, $E_{S 1(t)}$ is the optical field at the output of the amplifier given by:

$$
E_{S 1}(t)=\sqrt{G} E_{S 0}(t)
$$

where $\mathrm{G}$ is the optical amplifier gain and $\mathrm{E}_{\mathrm{S} 0}(\mathrm{t})$ is the optical field at the output of the modulator, previously defined in Eq. (11).

Finally, $E_{S_{2}}(t)$ is the field at the output of the filter. This means that this field will go through the photodetector following the expression:

$$
E_{S 2}(t)=E_{S 1}(t) * h_{0}(t)
$$

where $h_{0}(t)$ is the impulse response corresponding to the arbitrary filter frequency response $\mathrm{H}_{0}\left(\omega_{0}\right)$. In this case, it should be considered that the response $\mathrm{h}_{0}(\mathrm{t})$ is the combination of two transfer functions of the two arbitrary filters employed in the MWP link:

$$
h_{0}(t)=h_{1}(t) * h_{2}(t)
$$

Thus, the field described in Eq. (13) can be defined as:

$$
E_{S 2}(t)=j \sqrt{G \alpha_{m z m} P_{i n}} \sum_{n=-\infty}^{\infty} B_{n} J_{n}\left(\frac{\phi_{r f}}{2}\right) H_{0}\left(\omega_{0}+n \Omega\right) e^{j\left(\omega_{0}+n \Omega\right) t}
$$

On the other hand, $\mathrm{E}_{\mathrm{ASE} 1}(\mathrm{t})$ is the ASE field generated due to an active element like the OA and it is defined as:

$$
E_{A S E 1}(t)=\sqrt{N \hbar \omega_{0} \partial \omega} \sum_{k=-M}^{M} e^{j\left[\left(\omega_{0}+k \partial \omega\right) t+\varphi_{k}\right]}
$$

where $\mathrm{N}$, which is the ASE photon number output from the amplifier that is added to the signal, is given by $\mathrm{N}=\mathrm{n}_{\mathrm{sp}}(\mathrm{G}-1)$ (it is derived from quantum theory [11]) and $\mathrm{n}_{\mathrm{sp}}$ is the population inversion factor, $\hbar$ is the Planck constant divided by $2 \pi, \omega_{0}$ is the central frequency, $\delta \omega$ is the frequency discretization interval for the ASE spectrum $\left(\mathrm{B}_{\mathrm{ASE}}=2 \mathrm{M} \delta \omega\right)$, $\mathrm{M}$ is the number of ASE modes and $\varphi_{\mathrm{k}}$ is a random phase corresponding to the frequency discretize $\mathrm{k}$. The total ASE noise field is assumed to be composed by the superimposition of $2 \mathrm{M}$ independent radiation modes at the optical frequency $\omega_{0}+k \delta \omega$ with a random phase $\varphi_{\mathrm{k}}$.

Assuming that the ASE field from Eq. (16) propagates through the optical filter, the $\mathrm{E}_{\mathrm{ASE}_{2}}(\mathrm{t})$ is calculated as: 


$$
E_{A S E 2}(t)=E_{A S E 1}(t) * h_{0}(t)
$$

Replacing Eq. (14) and Eq. (16) in Eq. (17), we can be obtain:

$$
E_{A S E 2}(t)=\sqrt{N \hbar \omega_{0} \partial \omega} \sum_{k=-M}^{M} H_{0}\left(\omega_{0}+k \partial \omega\right) e^{j\left[\left(\omega_{0}+k \partial \omega\right) t+\varphi_{k}\right]}
$$

Afterward, photodetectors transfer intensity variations in the optical power to amplitude variations in the output photocurrent. So, then the instantaneous photocurrent $i(t)$ at the output of the photodetector is given by:

$$
i(t)=\mathfrak{R}\left[E_{S 2}(t)+E_{A S E 2}(t)\right]^{2}
$$

where the photodetector responsivity is defined like $\mathfrak{R}=\eta \mathrm{e} / \mathrm{h} \omega_{0}, \eta$ is the photodiode quantum efficiency and e is the electron charge constant. Developing Eq. (19), we can obtain:

$$
i(t)=\underbrace{\mathfrak{R}\left|E_{S 2}(t)\right|^{2}}_{i_{S 2}(t)}+\underbrace{\mathfrak{R}\left|E_{A S E 2}(t)\right|^{2}}_{i_{s p-s p}(t)}+\underbrace{\mathfrak{R}\left|E_{A S E 2}^{*}(t) E_{S 2}(t)+E_{S 2}^{*}(t) E_{A S E 2}(t)\right|}_{i_{s i p-s p}(t)}
$$

The first term is the detected signal $\left(\mathrm{i}_{\mathrm{S} 2}(\mathrm{t})\right)$, the second and the third terms are the spontaneousspontaneous and the signal-spontaneous beating noise currents generated respectively. From these definitions, the noise formulas will be developed in the next sections.

\subsubsection{Signal-spontaneous beat noise}

From the third term of Eq. (20), we can obtain the signal-spontaneous photocurrent as:

$$
i_{\text {sig-sp }}(t)=\mathfrak{R}\left[E_{A S E 2}^{*}(t) E_{S 2}(t)+E_{S 2}^{*}(t) E_{A S E 2}(t)\right]
$$

Developing Eq. (21), the time average of the squared photocurrent is given by:

$$
\left\langle i_{s i g-s p}^{2}(t)\right\rangle=2 \Re^{2} \alpha_{m z m} G P_{i n} N \hbar \omega_{0}\left|H_{0}\left(\omega_{0}\right)\right|^{2} B_{0, e q} \sum_{n=-\infty}^{\infty}\left|B_{n}\right|^{2} J_{n}^{2}\left(\frac{\phi_{r f}}{2}\right)\left|H_{0}\left(\omega_{0}+n \Omega\right)\right|^{2}
$$

The dominant term is for $\mathrm{n}=0$. Then, neglecting the rest of the contribution, we obtain:

$$
\left\langle i_{s i g-s p}^{2}(t)\right\rangle=2 \mathfrak{R}^{2} \alpha_{m z m} G P_{i n} N \hbar \omega_{0}\left|H_{0}\left(\omega_{0}\right)\right|^{4} B_{0, e q} \sin ^{2}\left(\frac{\phi_{d c}}{2}\right)
$$

Using the time average of the squared photocurrent calculated in Eq. (23) and the mean squared of the photocurrent $\left(<i_{\text {sig-sp }}(t)>^{2}\right)$, which is zero in this case, we can compute the variance as:

$$
\left\langle\sigma_{\text {sig-sp }}^{2}\right\rangle=\left\langle i_{\text {sig-sp }}^{2}(t)\right\rangle-\left\langle i_{\text {sig-sp }}(t)\right\rangle^{2}=\eta^{2} \alpha_{m z m} G I_{N} I_{S}\left(1-\cos \left(\phi_{d c}\right)\right)\left|H_{0}\left(\omega_{0}\right)\right|^{2}
$$

Then, the noise current $\left(\mathrm{I}_{\mathrm{N}}\right)$ and the signal current ( $\left.\mathrm{I}_{\mathrm{S}}\right)$ are given by Eq. (25) and Eq. (26) respectively. 


$$
\begin{gathered}
I_{N}=e N \int_{-\infty}^{\infty}\left|H_{0}\left(\omega_{0}\right)\right|^{2} \partial \omega \\
I_{S}=\frac{e P_{i n}}{\hbar \omega_{0}}
\end{gathered}
$$

where $\mathrm{I}_{\mathrm{N}}$ will be limited by the equivalent bandwidth of the optical filter used.

Subsequently, the noise power density distribution (in watts) is calculated integrating the spectral distribution derived in Eq. (24), along a uniform equivalent arbitrary filter with a single-side bandwidth given by $\mathrm{B}_{\mathrm{o}, \mathrm{eq}} / 2$. Moreover, it is multiplied by $\mathrm{R}_{\mathrm{OUT}}$ obtaining [11]:

$$
N_{s i g-s p}(f)=\frac{2\left\langle\sigma_{s i g-s p}^{2}\right\rangle R_{O U T}}{B_{o, e q}}=\frac{2 \eta^{2} \alpha_{m z m} G I_{N} I_{S}\left(1-\cos \left(\phi_{d c}\right)\right) R_{O U T}}{B_{o, e q}}\left|H_{0}\left(\omega_{0}\right)\right|^{2}
$$

\subsubsection{Spontaneous -spontaneous beat noise}

A similar derivation can be performed for the spontaneous-spontaneous current.

$$
i_{s p-s p}(t)=\Re\left|E_{A S E 2}(t)\right|^{2}
$$

From Eq. (28), the variance can be computed as:

$$
\left\langle\sigma_{s p-s p}^{2}\right\rangle=\eta^{2} I_{N}^{2}\left|H_{0}\left(\omega_{o}\right)\right|^{2}
$$

Finally, the noise power density distribution is defined as:

$$
N_{s p-s p}(f)=\frac{2 \eta^{2} I_{N}^{2} R_{O U T} M_{s p}}{B_{e q, o}}\left|H_{0}(\omega)\right|^{2}
$$

where, $\mathrm{M}_{\mathrm{sp}}$ accounts for the number of spontaneous emission modes $\left(\mathrm{M}_{\mathrm{sp}}\right.$ is either 1 or 2 depending on whether one or the two polarization states of spontaneous emission are considered) [11].

\subsubsection{Spontaneous-shot noise}

For spontaneous-shot noise, we can use the second term of Eq. (20) to calculate its variance which is given by:

$$
\left\langle\sigma_{s p, s h o t}^{2}\right\rangle=2 e\left\langle i_{s p-s p}(t)\right\rangle
$$

Using Eq. (31) and Eq. (28), the noise power density distribution of shot noise due to amplified spontaneous emission power is calculated as:

$$
N_{s p-s h o t}(f)=\left\langle\sigma_{s p, s h o t}^{2}\right\rangle R_{O U T}=2 e \eta I_{N} M_{s p} R_{O U T}
$$

\subsubsection{Signal-shot noise}

Following Eq. (20) and $\mathrm{Eq}(15)$, the detected signal is given by: 


$$
\begin{aligned}
& i_{S 2}(t)=\mathfrak{R}\left|E_{S 2}(t)\right|^{2}= \\
& =\mathfrak{R} G \alpha_{m z m} P_{i n} \sum_{n=-\infty}^{\infty} \sum_{m=-\infty}^{\infty} B_{n} B_{m}^{*} J_{n}\left(\frac{\phi_{r f}}{2}\right) J_{m}\left(\frac{\phi_{r f}}{2}\right) H_{0}\left(\omega_{0}+n \Omega\right) H_{0}^{*}\left(\omega_{0}+m \Omega\right) e^{j(n-m) \Omega t}
\end{aligned}
$$

From Eq. (33), the DC component can be computed in the case of $\mathrm{m}=\mathrm{n}$ and assuming that the dominant term is for $\mathrm{n}=0$.

$$
i_{d c}=\eta G I_{S} \alpha_{m z m} \sin ^{2}\left(\frac{\phi_{d c}}{2}\right)\left|H\left(\omega_{o}\right)\right|^{2}
$$

Then, using Eq. (34), signal-shot noise spectral power density will be:

$$
N_{\text {sig-shot }}(f)=2 e\left\langle i_{d c}(t)\right\rangle R_{\text {OUT }}=e \eta G \alpha_{m z m} I_{S}\left(1-\cos \left(\phi_{d c}\right)\right) R_{\text {OUT }}\left|H_{0}\left(\omega_{o}\right)\right|^{2}
$$

\subsubsection{Input and output thermal noise}

The output thermal noise is originated from the RF and electronic driving circuitry after the photodiode. The output noise spectral power density is:

$$
N_{\text {thermal }, o}(f)=k_{B} T
$$

where $\mathrm{k}_{\mathrm{B}}$ is the Boltzmann constant and $\mathrm{T}$ is the temperature in Kelvin degrees.

In the same way, the input thermal noise originated from RF and electronic driving circuitry of the modulator. So, the input noise spectral power density is given by:

$$
N_{\text {thermal }, i-p h d}(f)=G_{R F} N_{\text {thermal }, i}(f)
$$

where $\mathrm{G}_{\mathrm{RF}}$ is the RF gain of the link defined in Eq. (1).

\subsubsection{Amplified arbitrary filtered microwave photonic links figures of merit}

Once we know all the noise spectral power densities generated in the link, we can use Eq. (2) to calculate the total relative intensity noise.

Then, from Eq. (2) the signal-spontaneous RIN can be derived using Eq. (27) and Eq. (34):

$$
R I N_{s i g-s p}=\frac{8 I_{N}}{I_{S} G \alpha_{m z m}\left(1-\cos \left(\phi_{d c}\right)\right)\left|H_{0}\left(\omega_{0}\right)\right|^{2} B_{o, e q}}
$$

In the same way, spontaneous-spontaneous RIN can be derived using Eq. (30) and Eq. (34):

$$
\operatorname{RIN}_{s p-s p}=\frac{8 I_{N}^{2} M_{s p}}{I_{S}^{2} G^{2} \alpha_{m z m}^{2}\left(1-\cos \left(\phi_{d c}\right)\right)^{2}\left|H_{0}\left(\omega_{0}\right)\right|^{2} B_{e q, 0}}
$$

Also, the spontaneous-shot RIN is calculated from Eq. (32) and Eq. (34): 


$$
\operatorname{RIN}_{s p-s h o t}=\frac{8 e I_{N} M_{s p}}{\eta G^{2} I_{s}^{2} \alpha_{m z m}^{2}\left(1-\cos \left(\phi_{d c}\right)\right)^{2}\left|H_{0}\left(\omega_{0}\right)\right|^{4}}
$$

Therefore, the signal-shot RIN can be expressed using Eq. (35) and Eq. (34).

$$
R I N_{\text {sig-shot }}=\frac{4 e}{\eta G I_{S} \alpha_{m z m}\left[1-\cos \left(\phi_{d c}\right)\right]\left|H_{0}\left(\omega_{0}\right)\right|^{2}}
$$

Lastly, the thermal output and input relative intensity noises are calculated using Eqs. (36) and (37) respectively and the DC the intensity component calculated in Eq. (34).

$$
\begin{gathered}
\operatorname{RIN}_{t h, o}=\frac{4 k_{B} T}{\eta^{2} G^{2} \alpha_{m z m}^{2} I_{S}^{2}\left(1-\cos \left(\phi_{d c}\right)\right)^{2}\left|H_{0}\left(\omega_{0}\right)\right|^{4} R_{\text {OUT }}} \\
\operatorname{RIN}_{t h, i}=\frac{k_{B} T \pi^{2} \sin ^{2}\left(\phi_{d c}\right) R_{i n}\left|H_{0}(\Omega)\right|^{2}}{16\left(1-\cos \left(\phi_{d c}\right)\right)^{2} V_{\pi}^{2}\left|H_{0}\left(\omega_{0}\right)\right|^{4}}
\end{gathered}
$$

where $\left|\mathrm{H}_{0}(\Omega)\right|^{2}=\left[\mathrm{H}_{0}\left(\omega_{0}\right) \mathrm{H}_{0}{ }^{*}\left(\omega_{0}+\Omega\right)+\mathrm{H}_{0}\left(\omega_{0}+\Omega\right) \mathrm{H}_{0}{ }^{*}\left(\omega_{0}\right)\right]$.

Finally, RIN ${ }_{\text {TOт }}$ is calculated in Eq. (44) as the addition of the six relative intensity noises Eqs. (38), (39), (40), (41), (42) and (43) obtained previously.

$$
\operatorname{RIN}_{\text {TOT }}=\operatorname{RIN}_{\text {sig-sp }}(f)+\operatorname{RIN}_{s p-s p}(f)+\operatorname{RIN}_{s p-\text { shot }}(f)+\operatorname{RIN}_{\text {thermal }, i}(f)+\operatorname{RIN}_{\text {thermal }, o}(f)+\operatorname{RIN}_{\text {sig-shot }}(f)
$$

So, the noise figure of the link can be expressed as:

$$
N F_{\text {TOT }}=N F_{\text {sig-sp }}(f)+N F_{s p-s p}(f)+N F_{s p-s h o t}(f)+N F_{\text {thermal }, i}(f)+N F_{\text {thermal }, o}(f)+N F_{\text {sig-shot }}(f)
$$

where, using Eq. (4) each NF source can be expressed as:

$$
\begin{gathered}
N F_{\text {sig-sp }}=\frac{128 V_{\pi}^{2} I_{N}\left(1-\cos \left(\phi_{d c}\right)\right)\left|H_{0}\left(\omega_{0}\right)\right|^{2}}{\pi^{2} R_{i n} G \alpha_{m z m} I_{S} \sin ^{2}\left(\phi_{d c}\right)\left|H_{0}(\Omega)\right|^{2} B_{e q, o} k_{B} T} \\
N F_{s p-s p}=\frac{128 V_{\pi}^{2} I_{N} M_{s p}\left|H_{0}\left(\omega_{0}\right)\right|^{2}}{\pi^{2} R_{i n} G^{2} \alpha_{m z m} I_{S}^{2} \sin ^{2}\left(\phi_{d c}\right)\left|H_{0}(\Omega)\right|^{2} B_{e q, o} k_{B} T} \\
N F_{s p-s h o t}=\frac{128 V_{\pi}^{2} I_{N} e M_{s p}}{\pi^{2} R_{i n} G^{2} \alpha_{m z m}^{2} \eta I_{S}^{2} \sin ^{2}\left(\phi_{d c}\right)\left|H_{0}(\Omega)\right|^{2} k_{B} T} \\
N F_{t h, o}=\frac{1}{G_{R F}}
\end{gathered}
$$




$$
\begin{gathered}
N F_{t h, i}=1 \\
N F_{\text {sig-shot }}=\frac{64 V_{\pi}^{2} e\left(1-\cos \left(\phi_{d c}\right)\right)\left|H_{0}\left(\omega_{0}\right)\right|^{2}}{\pi^{2} R_{i n} G \alpha_{m z m} \eta I_{S} \sin ^{2}\left(\phi_{d c}\right)\left|H_{0}(\Omega)\right|^{2} k_{B} T}
\end{gathered}
$$

For the dynamic range calculation, we need the RF power at the output of the link. It can be obtained from the detected photocurrent (Eq. (33)), which delivers an electrical power to the output impedance $\mathrm{R}_{\mathrm{OUT}}$ as:

$$
P_{R F_{\text {OUT }}}(\Omega)=\frac{\left\langle\left|i_{S 2}(\Omega, t)\right|^{2}\right\rangle R_{\text {OUT }}}{2}=\frac{I_{d c}^{2} \sin ^{2}\left(\phi_{d c}\right) \phi_{r f}^{2}\left|H_{0}(\Omega)\right|^{2} R_{\text {OUT }}}{8}
$$

The expression for the photodetected RF power relative to the $\mathrm{IMD}_{2}$ term at the angular frequency $\Omega_{1} \pm \Omega_{2}$ and relative to IMD 3 term at $2 \Omega_{1} \pm \Omega_{2}$ are obtained as Eqs. (53) and (54) respectively.

$$
\begin{aligned}
& P_{R F_{\text {OUT }}}\left(\Omega_{1} \pm \Omega_{2}\right)=\frac{I_{d c}^{2}\left|I M D_{2}\right|^{2} \phi_{r f}^{4} R_{\text {OUT }}}{128} \\
& P_{R F_{\text {OUT }}}\left(2 \Omega_{1} \pm \Omega_{2}\right)=\frac{I_{d c}^{2}\left|I M D_{3}\right|^{2} \phi_{r f}^{6} \sin ^{2}\left(\phi_{r f}\right) R_{\text {OUT }}}{8192}
\end{aligned}
$$

where $\mathrm{IMD}_{2}=\left[\mathrm{IMD}_{21}-\mathrm{IMD}_{22}+\cos \left(\phi_{\mathrm{dc}}\right)\left(\mathrm{IMD}_{21}+\mathrm{IMD}_{22}\right)\right]$ and $\mathrm{IMD}_{21}$ and $\mathrm{IMD}_{22}$ are given by Eq. (55) and Eq. (56) respectively and $\mathrm{IMD}_{3}$ is given by Eq.(57):

$$
\begin{aligned}
& I M D_{21}\left(\omega_{0}, \Omega_{1}, \Omega_{2}\right)=\left[H_{0}\left(\omega_{0}+\Omega_{1}\right) H_{0}^{*}\left(\omega_{0}+\Omega_{2}\right)+H_{0}\left(\omega_{0}-\Omega_{2}\right) H_{0}^{*}\left(\omega_{0}-\Omega_{1}\right)\right] \\
& I M D_{22}\left(\omega_{0}, \Omega_{1}, \Omega_{2}\right)=\left[H_{0}\left(\omega_{0}\right) H_{0}^{*}\left(\omega_{0}-\Omega_{1}+\Omega_{2}\right)+H_{0}\left(\omega_{0}+\Omega_{1}-\Omega_{2}\right) H_{0}^{*}\left(\omega_{0}\right)\right] \\
& I M D_{3}\left(\omega_{0}, \Omega_{1}, \Omega_{2}\right)=\left[\begin{array}{l}
H_{0}\left(\omega_{0}\right) H^{*}\left(\omega_{0}-2 \Omega_{1}+\Omega_{2}\right)+H_{0}^{*}\left(\omega_{0}\right) H\left(\omega_{0}+2 \Omega_{1}-\Omega_{2}\right)+ \\
+H_{0}\left(\omega_{0}+2 \Omega_{1}\right) H^{*}\left(\omega_{0}+\Omega_{2}\right)+H_{0}\left(\omega_{0}-\Omega_{2}\right) H^{*}\left(\omega_{0}-2 \Omega_{1}\right)
\end{array}\right]
\end{aligned}
$$

Using Eqs. (52) and (53) we can calculate the $\mathrm{OIP}_{2}$ and $\mathrm{OIP}_{3}$ is obtained from Eqs. (52) and (54). It should be noted that these values of $\mathrm{OIP}_{2}$ and $\mathrm{OIP}_{3}$ defined in Eqs. (58) and (59) respectively remain unaltered for all the amplified link configurations.

$$
\begin{aligned}
& O I P_{2}=\frac{2 R_{\text {OUT }} I_{d c}^{2} \sin ^{4}\left(\phi_{d c}\right)\left|H_{0}(\Omega)\right|^{4}}{\left|I M D_{2}\right|^{2}} \\
& O I P_{3}=\frac{4 R_{O U T} I_{d c}^{2} \sin ^{2}\left(\phi_{d c}\right)\left|H_{0}(\Omega)\right|^{3}}{\left|I M D_{3}\right|}
\end{aligned}
$$


Finally, using Eqs. (6) and (7) the SFDR can be calculated with the OIPs and $\mathrm{N}_{\text {Tот }}$ expressions derived above.

$$
\begin{gathered}
S F D R_{2}=\left[\frac{O I P_{2}}{N_{\text {TOT }}}\right]^{1 / 2}=\sqrt{\frac{2 R_{\text {OUT }} I_{d c}^{2} \sin ^{4}\left(\phi_{d c}\right)\left|H_{0}(\Omega)\right|^{4}}{\left|I M D_{2}\right|^{2} N_{T O T}}} \\
S F D R_{3}=\left[\frac{O I P_{3}}{N_{T O T}}\right]^{2 / 3}=\sqrt[3]{\left(\frac{4 R_{O U T} I_{d c}^{2} \sin ^{2}\left(\phi_{d c}\right)\left|H_{0}(\Omega)\right|^{3}}{\left|I M D_{3}\right| N_{T O T}}\right)^{2}}
\end{gathered}
$$

It should be pointed out that the model Eqs. (12) - (62) converge exactly to those derived in [6] for the case of passive arbitrary MWP links, i.e. when $\mathrm{G}=1$ (no amplification).

\subsection{In-line link/system}

In contrast with the power amplifier case, if the amplifier is placed in-line (Fig. 3), the noise is only filtered by the second filter. Therefore, the theoretical derivations for these MWP links/systems are similar to those of power amplification except that the optical filter transfer function used in Eq. (25) is given now by $\mathrm{H}_{0}\left(\omega_{0}\right)=\mathrm{H}_{2}\left(\omega_{0}\right)$. It means that is only in relation to amplifier noise where $\mathrm{H}_{1}\left(\omega_{0}\right)$ has no effect.

The FOMs for this case can be calculated using the same generic expressions derived in the previous section taking into account the prescription given in the former paragraph.

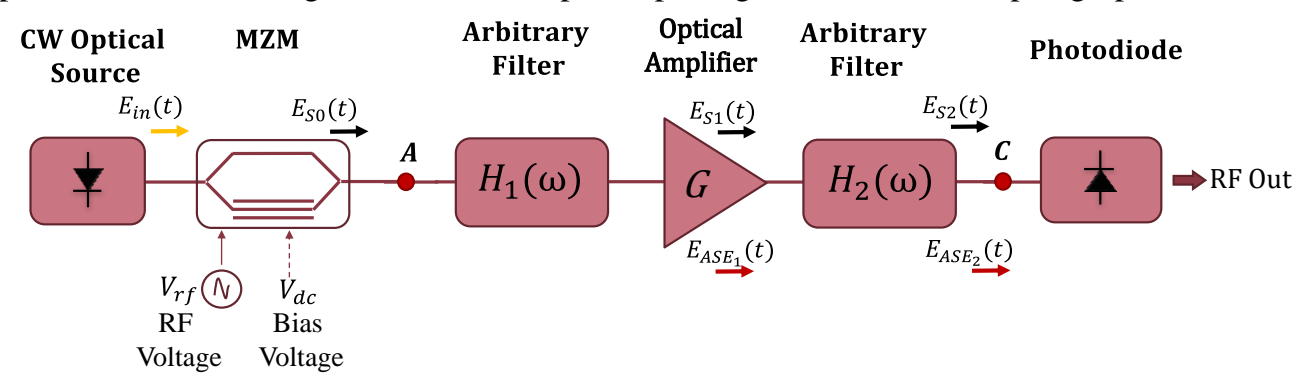

Fig. 3. In-line-amplified filtered MWP link/system for the analysis.

\subsection{Pre-amplified link/system}

In a similar way, when the amplifier is after the filter (Fig. 4) the noise generated due to the amplifier is not filtered.

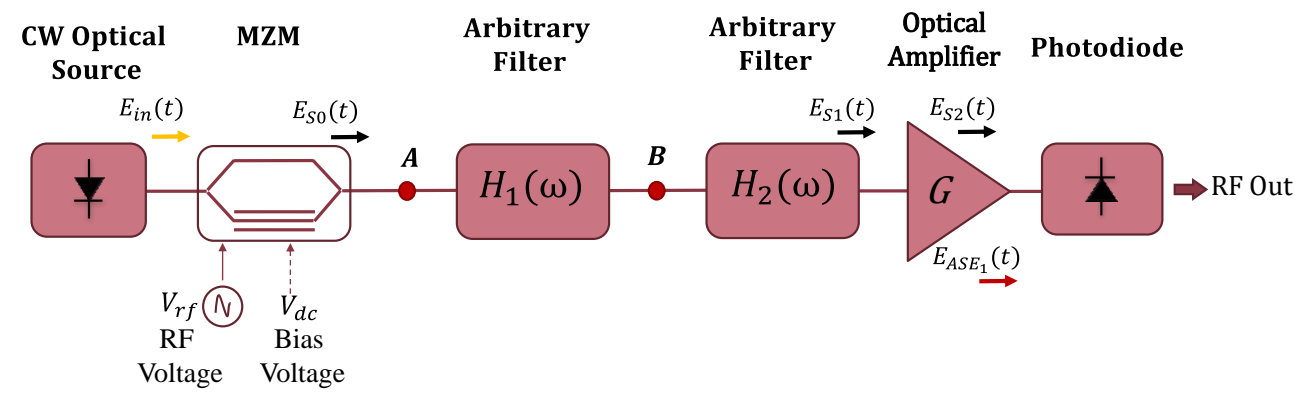

Fig. 4. Pre-amplified filtered MWP link/system for this analysis. 
Hence, applying an equivalent flat-top optical filter response to the arbitrary filter, we can redefine noise current from Eq. (25) as:

$$
I_{N}=e N B_{o, e q}
$$

where $B_{0, \text { eq }}$ is the equivalent optical filter bandwidth defined in [11]. Finally, the signal current is defined following Eq. (26) and the FOM can be analyzed using the generic expressions derived in section 2.2.6.

\section{Application to a microwave photonics link}

As a proof, we have applied the analytical model to evaluate two interesting but quite different applications. We employed Python programming language to analyze both amplified arbitrary filtered Microwave Photonic links and integrated MWP systems. The model achieves complete flexibility and versatility to obtain the impact of the components on the overall system/link performance.

\subsection{Optical Fiber MWP Links}

The first example corresponds to a MWP optical fiber link, implemented by two sections of dispersive Single Mode Fibers (SMF) with a total length of 35Km (Fig. 5). For this example, we set the optical amplifier gain and the noise figure to $13 \mathrm{~dB}$ and $6 \mathrm{~dB}$ respectively. These values are based on typical specifications of a commercial EDFA.

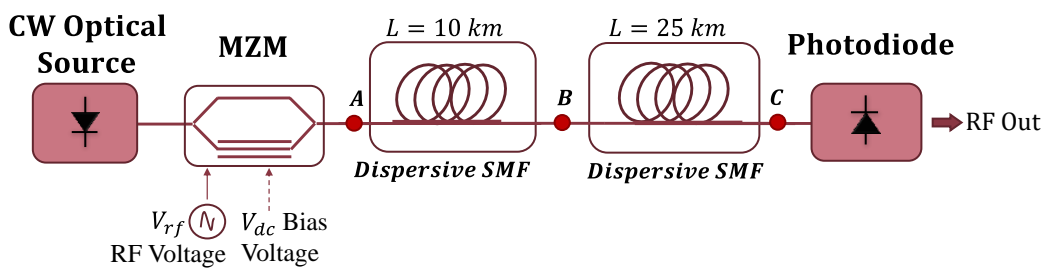

Fig. 5. Schematic of an amplifier filtered MWP system (point A power-amplified, point B in-line amplified and point C pre-amplified) optical link of $35 \mathrm{~km}$.

We set two tones at microwave frequencies of $\mathrm{f}_{1}=4.1 \mathrm{GHz}$ and $\mathrm{f}_{2}=4.2 \mathrm{GHz}$, at the input of the intensity modulator, to carry out the dynamic range evaluation. The transfer functions of the two dispersive SMF fibers elements are given by $\mathrm{H}_{1}\left(\omega_{0}\right)=\mathrm{H}_{2}\left(\omega_{0}\right)=\mathrm{e}^{-\mathrm{\alpha L} / 2} \mathrm{e}^{-\mathrm{j} \beta \mathrm{L}}$ (see Fig. 6), where $\alpha$ is the fiber optical loss $(0.2 \mathrm{~dB} / \mathrm{km}), \mathrm{L}$ is the length of each fiber spool and $\beta$ is the propagation constant. Here, the fiber is divided in two spools with lengths $\mathrm{L}=10 \mathrm{~km}$ and $\mathrm{L}=$ $25 \mathrm{~km}$ (the total length of the dispersive stage is $35 \mathrm{~km}$ ) and the equivalent optical filter bandwidth is $200 \mathrm{GHz}[10,11]$. Finally, a photodetector is placed at the end of the link. The following parameters have fixed values: the modulator half-way voltage $\mathrm{V}_{\pi}=5 \mathrm{~V}$, quadrature MZM bias point $\phi_{\mathrm{dc}}=\pi / 2$, optical input power $\mathrm{P}_{\mathrm{in}}=7 \mathrm{dBm}$, responsivity of photodetector $\Re=$ $0.6 \mathrm{~A} / \mathrm{W}$, modulator insertion losses $\alpha_{\mathrm{mzm}}=8 \mathrm{~dB}$, photodiode quantum efficiency $\eta=1$ and input and output loads $\mathrm{R}_{\text {in }}=\mathrm{R}_{\text {out }}=50 \Omega$. 


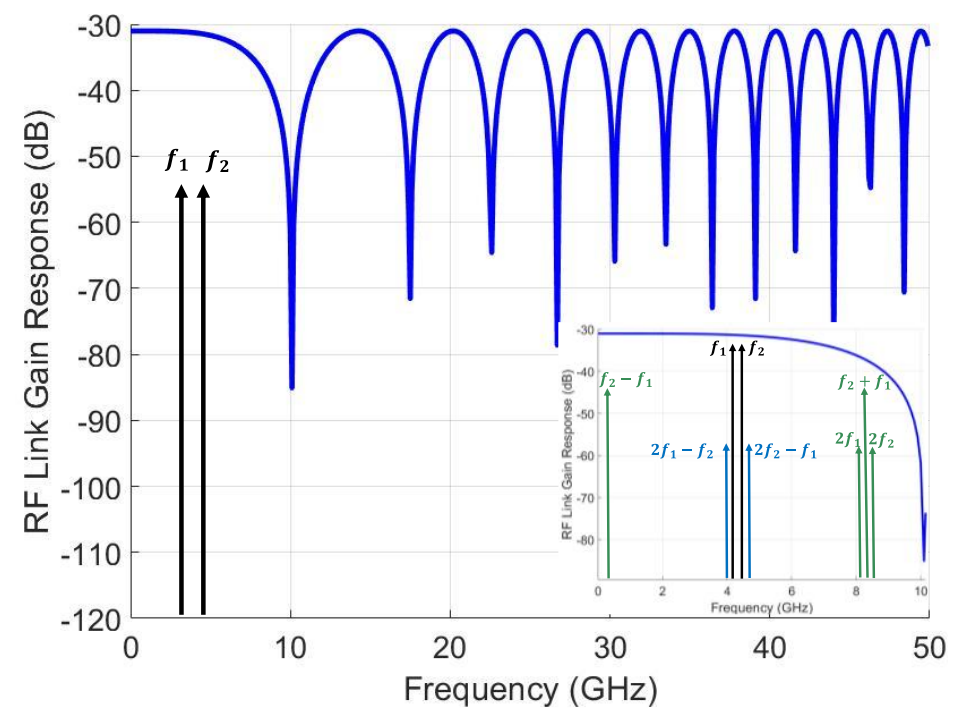

Fig. 6. Analytical simulation of $\mathrm{G}_{\mathrm{RF}}$ response in the dispersive link for intensity modulation with $10 \mathrm{~km}$ and $25 \mathrm{~km}$ SMF elements. The optical amplifier gain and the noise figure is set to $13 \mathrm{~dB}$ and $6 \mathrm{~dB}$ respectively. The inset shows the $\mathrm{G}_{\mathrm{RF}}$ response with the location of $\mathrm{f}_{1}, \mathrm{f}_{2}$ and the distortion terms (blue: 3rd order and green: 2 nd order).

Table 1 shows a comparison of the FOMs values obtained from the model of the optical fiber MWP link in each case of amplification.

Table 1. Simulation results from power, pre and in-line amplifier link.

\begin{tabular}{|c|c|c|c|c|c|}
\hline Link Scheme & $\begin{array}{c}\mathbf{S F D R}_{2} \\
\left(\mathrm{dBm} \cdot \mathrm{Hz}^{1 / 2}\right)\end{array}$ & $\begin{array}{c}\mathbf{S F D R}_{\mathbf{3}} \\
\left(\mathrm{dBm} \cdot \mathrm{Hz}^{2 / 3}\right)\end{array}$ & $\begin{array}{c}\mathbf{N}_{\text {TOT }} \\
(\mathrm{dBm} / \mathrm{Hz})\end{array}$ & $\begin{array}{c}\mathbf{R I N}_{\text {ToT }} \\
(\mathrm{dB} / \mathrm{Hz})\end{array}$ & $\begin{array}{c}\text { Gain } \\
(\mathbf{d B})\end{array}$ \\
\hline Power Amplifier & 83.04 & 99.1 & -153.59 & -139.05 & -31.31 \\
\hline In-line Amplifier & 82.07 & 97.81 & -151.66 & -137.13 & -31.31 \\
\hline Pre-Amplifier & 79.59 & 94.5 & -146.70 & -132.18 & -31.31 \\
\hline
\end{tabular}

As it can be extracted from Table 1, the total noise spectral power density, the relative intensity noise and the distortion terms improve when the amplifier is set before the filter (power amplification scheme) compared to the other schemes.

Moreover, although adding an amplifier to the link always increases the spectral noise density and the RIN, if it is positioned before the optical filter, some of the amplification related noises are mitigated by the filtering stage as we can see in Fig. 7. This fact contrasts with the pre-amplified link, where the spontaneous contributions of the amplifier are not filtered. Hence, if we are considering that the amplifier is working on linear gain regime (i.e. unsaturated and amplifying signals with time variations shorter than the dynamic gain time constant) a lower signal degradation will be obtained if the amplifier is placed before the filter. Finally, it is shown that if power amplification cannot be provided, the in-line amplifier configuration provides a significantly good performance, since it offers an improvement of the signal with partial noise filtering, compared with post amplification scheme. 


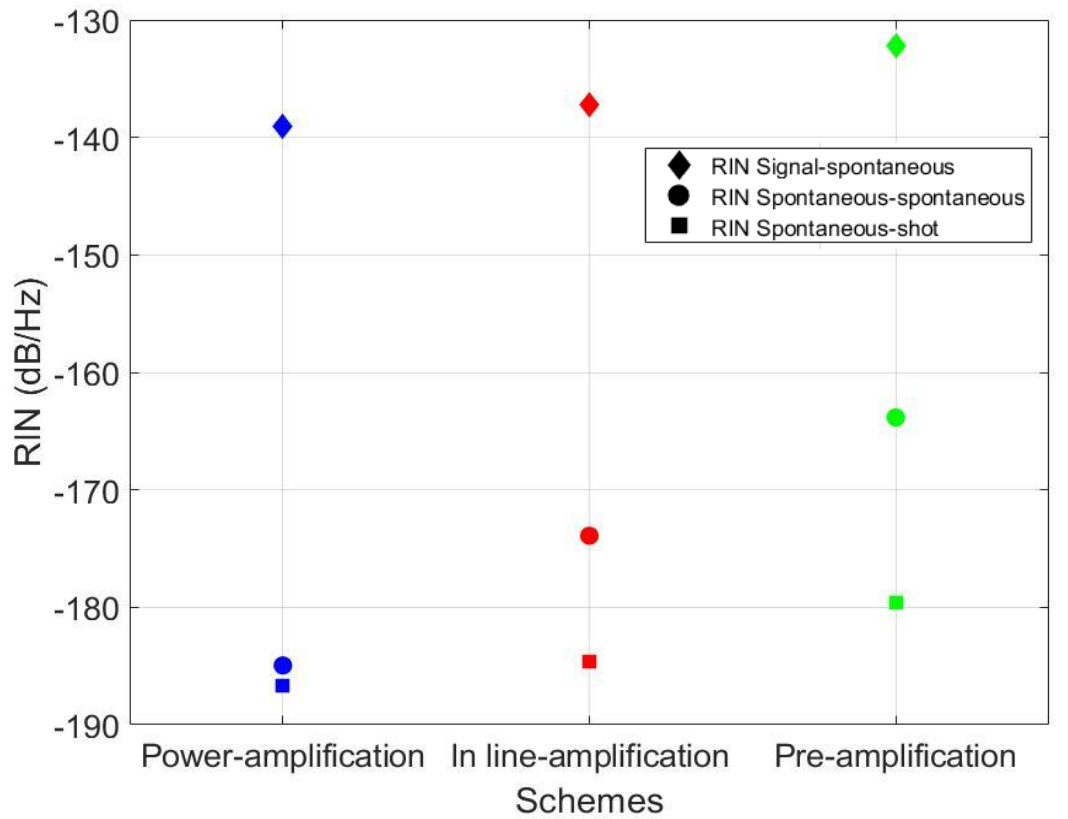

Fig. 7. Comparison of RIN levels for each link scheme (power-amplification, inline-amplification and preamplification) obtained after applying the analytical model derived in this work.

The versatility of the analytical model derived in this paper also allows for a deep analysis of the link/system parameters on the overall system performance. As a demonstration, we have run a parametric simulation study to evaluate the evolution of the link FOMs for the power-amplified case as a function of the amplifier's gain (and therefore its noise figure) and of the input power of the optical source. Results are shown in Fig. 8.

In the ranges considered, increasing the amplifier gain and the optical input power, a direct improvement of the link performance is obtained in terms of gain, noise figure and distortion.

However, $\mathrm{N}_{\mathrm{TOT}}$ gets slightly worse due to spontaneous emission created by the amplifier. It should be highlighted here that the optical input power of the laser should not saturate the amplifier. In this example, we have neglected gain saturation, although it can be added to the model by loading a typical saturation curve. Specifically, in terms of gain, we can see that by increasing the amplifier gain, an improvement of the MWP link gain is obtained, following a squared relation. 
(a)

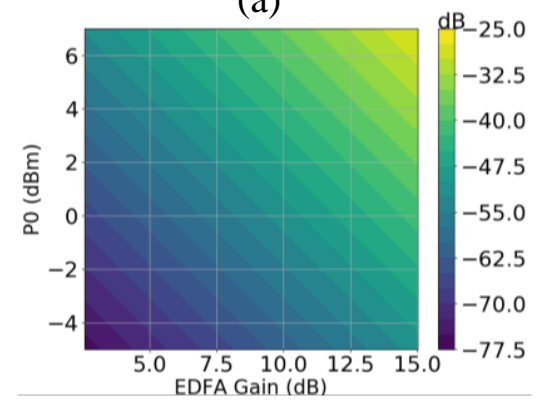

(c)

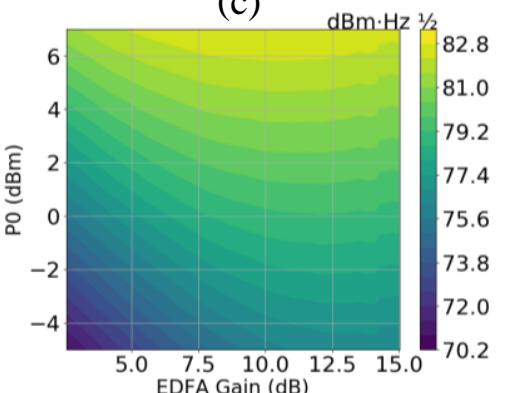

(e)

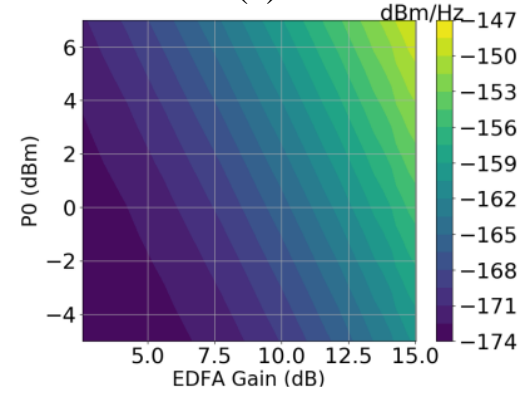

(b)

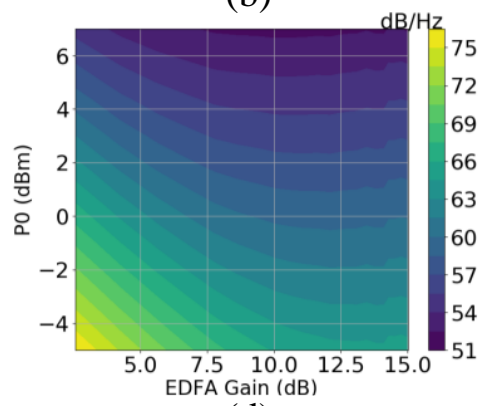

(d)

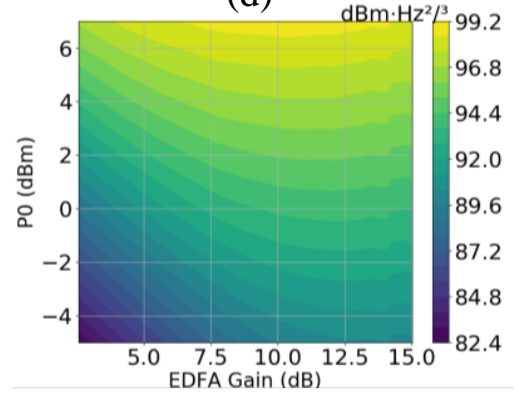

(f)

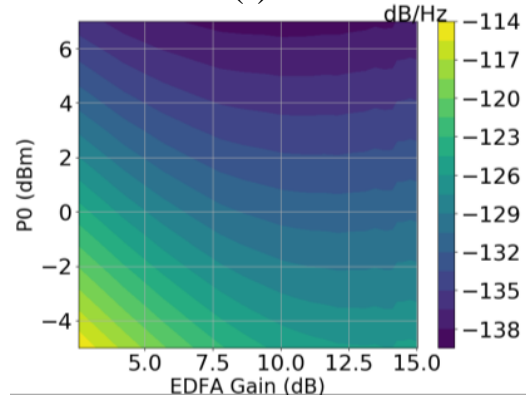

Fig. 8. Scheme MWP power-amplification long optical fiber link (a) gain, (b) Noise figure, (c)SFDR ${ }_{2},(d) \mathrm{SFDR}_{3}$, (e) Total noise spectral power density and (f) Relative intensity noise analysis.

\subsection{Microwave photonics system with a reconfigurable photonic waveguide mesh} filter

Again, the adaptability of the model makes it applicable to flexible and reconfigurable MWP systems. As a representative example, we consider a MWP system where a reconfigurable filter is inserted between the source and the detector (Fig. 9).

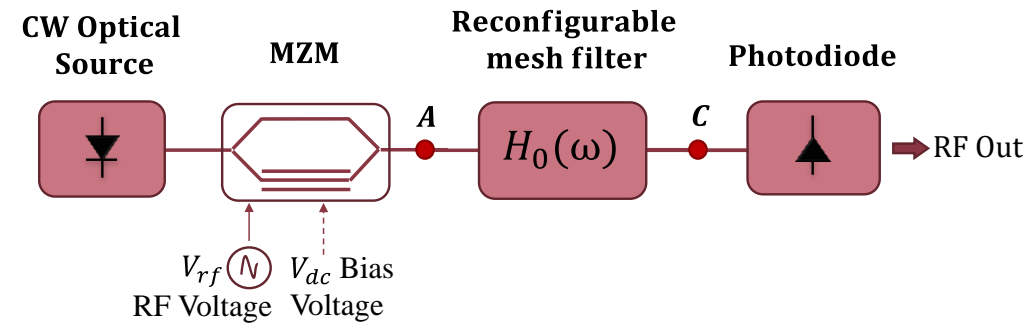

Fig. 9. Schematic of a MWP system with a reconfigurable photonic waveguide mesh filter (point A power-amplified, and point $\mathrm{C}$ pre-amplified). 
In this case, the optical subsystem implements a cascade MZI lattice filter of third order (Fig. 10. (a)), which is synthesized with a programmable hexagonal optical processor (Fig. 10. (b)) $[13,14]$. Such processor is based on a two-dimensional photonic waveguide mesh created by the interconnection of Programmable Unit Cells (PUC). Employing this type of reconfigurable optical core, we can synthetize a wide variety of photonic circuits and MWP applications [13].

(a)

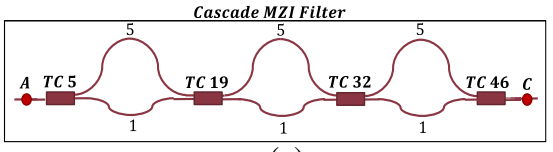

(c) (b)

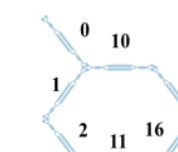

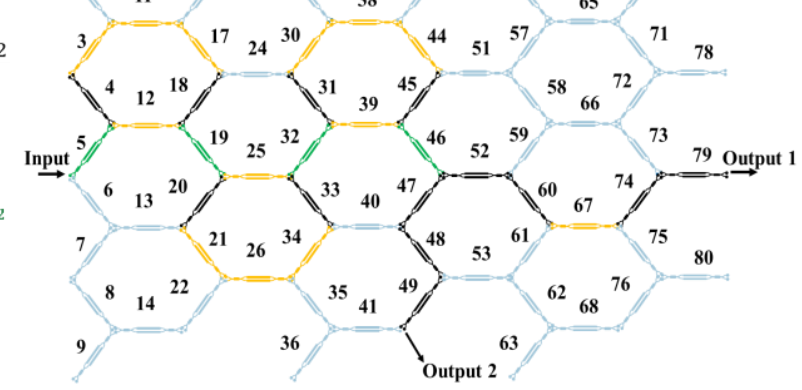

Fig. 10 (a) Schematic of the equivalent circuit that has been synthesized with the mesh (b) Filter synthesized with the reconfigurable mesh core (c) Filter response vs. normalized frequency with the location of $\mathrm{f}_{1}=19 \mathrm{GHz}, \mathrm{f}_{2}=21 \mathrm{GHz}$ and the distortion terms (blue: 3rd order and green: 2 nd order).

We have computed the lattice filter frequency response (Fig. 10. (c)) of the waveguide mesh arrangement by employing the analytical model derived in [14]. In the simulation, we have considered that the insertion loss of each unit cell is $0.3 \mathrm{~dB}$, and the insertion loss of the chip ports is $1.5 \mathrm{~dB}$ each. After feeding the optical response to our model, we have considered two locations (point A power-amplified and point $\mathrm{C}$ pre-amplified (Fig. 10 (a)) to place a semiconductor optical amplifier (Gain $=13 \mathrm{~dB}$ and noise figure $=6 \mathrm{~dB}[15]$ ) and evaluated the behaviour of the link performance.

The FOMs of a MWP system including this lattice filter and applying our model are show in Table 2. It is observed that the power-amplification case gives a much better noise figure than the pre-amplification case. This is because the effect of the loss introduced by the filter is directly proportional to the variation produced in the noise of the link. We can see that in both cases, the optical amplifier compensates the optical loss but the overall RF Gain remains below $-30 \mathrm{~dB}$. This analysis motivates the insertion of SOAs as high-performance building blocks in Field-Programmable Photonic Gate Arrays [16] and in application specific microwave photonic integrated circuits.

Table 2. Analytical results from power and pre amplifier system.

\begin{tabular}{|c|c|c|c|c|c|c|}
\hline Link Scheme & $\begin{array}{c}\mathbf{S F D R}_{\mathbf{2}} \\
\left(\mathrm{dBm} \cdot \mathrm{Hz}^{1 / 2}\right)\end{array}$ & $\begin{array}{c}\mathbf{S F D R}_{\mathbf{3}} \\
\left(\mathrm{dBm} \cdot \mathrm{Hz}^{2 / 3}\right)\end{array}$ & $\begin{array}{c}\mathbf{N}_{\text {TOT }} \\
(\mathrm{dBm} / \mathrm{Hz})\end{array}$ & $\begin{array}{c}\text { RINTot } \\
(\mathrm{dB} / \mathrm{Hz})\end{array}$ & $\begin{array}{c}\text { NF Link } \\
(\mathrm{dB})\end{array}$ & $\begin{array}{c}\text { Gain } \\
(\mathrm{dB})\end{array}$ \\
\hline Power Amplifier & 85.64 & 100.50 & -159.50 & -140.96 & 49.45 & -35.01 \\
\hline Pre-Amplifier & 80.23 & 93.28 & -148.68 & -130.19 & 60.27 & -35.01 \\
\hline
\end{tabular}

Finally, we ran a parametric analysis of the power-amplification scheme (Fig. 11), where one can observe a similar trend to the one shown for the optical fiber link. 
(a)

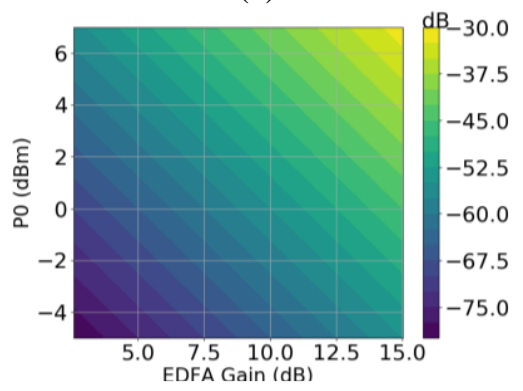

(c)

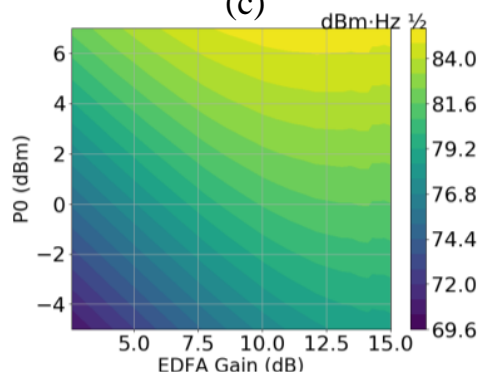

(e)

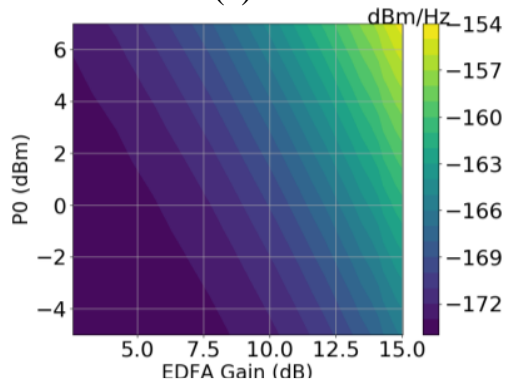

(b)

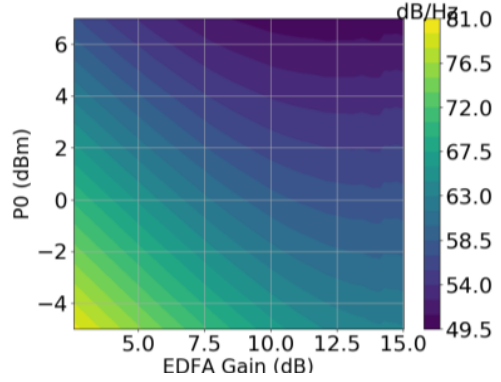

(d)

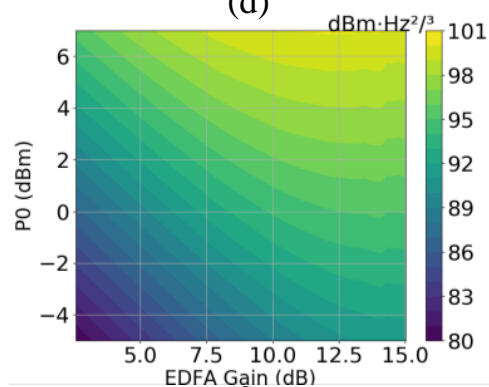

(f)

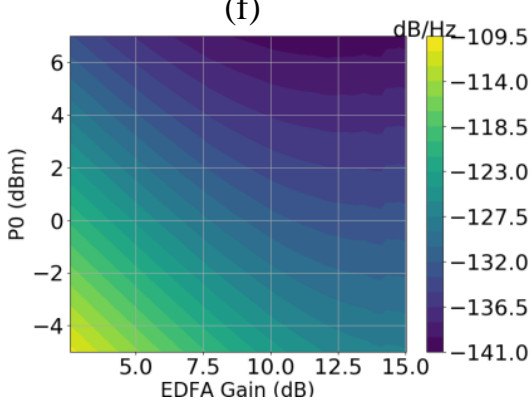

Fig. 11. Power-amplification scheme MWP system with a reconfigurable photonic waveguide mesh filter (a) Gain,

(b) Noise figure, (c)SFDR 2 , (d) $\mathrm{SFDR}_{3}$, (e) Total noise spectral power density and (f) Relative intensity noise analysis.

\subsection{Discussion}

Microwave photonics systems have proven remarkable performance. However, their application in real-world scenarios is limited by reduced RF gains, noise figures and system linearity. Improving RF Gain through the insertion of optical amplifiers is straightforward but impacts on the noise of the overall system. The model developed in Section 2, concise in Eqs. (38)-(51) and applied to two different examples in Section 3 enables the parametric study during the design and control of microwave photonic systems/links. It provides analytical expressions for a generic and simple evaluation of the main figures of merit characterizing amplified arbitrary filtered MWP links and systems and can be employed as a powerful analytical tool for prediction, optimization, evaluation, and redesign tasks.

Moreover, the surge of flexible and reconfigurable MWP circuits calls for a modelling tool that can cope with the system flexibility. The model reported here enables a versatile evaluation of the position of the amplifier and its application to arbitrary systems, as long as they can be defined as a chain of passive and linear spectral subsystems. 


\section{Conclusion}

We developed a model of amplified complex microwave photonic systems, which is adaptive to arbitrary system configurations. In addition, it enables the analysis and impact of each link/system parameters and the optical amplifier position. This versatile model can be employed to evaluate the performance of arbitrary MWP links and systems.

\section{Acknowledgments}

The authors acknowledge financial support by the ERC ADG-2016 UMWP-Chip, ERC-POC2019 FPPAs, the Generalitat Valenciana PROMETEO 2017/017 research excellency award, the COST Action CA16220 EUIMWP and the Juan de la Cierva Postdoctoral grant.

\section{Disclosures}

The authors declare no conflicts of interest.

\section{References}

1. J. Yao, "Microwave photonics," J. Lightw. Technol. 27(3), 314-335, (2009).

2. J. Capmany and D. Novak, "Microwave photonics combines two worlds," Nature Photon. 1(6), 319-330 (2007).

3. T. Berceli and P. R. Herczfeld, "Microwave photonics-A historical perspective," IEEE Trans. Microwave Theory Tech., 58 (11), 2992-3000 (2010).

4. C. H. Cox III, Analog Photonic Links: Theory and Practice, (Cambridge University Press, 2004).

5. D. Marpaung, J. Yao, J. Capmany, "Integrated microwave Photonics," Nature Photonics 13, 80-90, (2019).

6. I. Gasulla, and J. Capmany, "Analytical model and figures of merit for filtered microwave photonic links," Opt. Express, 19, 19758-19774 (2011)

7. Y. Liu, A. Choudhary, D. Marpaung, \& B. J. Eggleton, "Integrated microwave photonic filters" Adv. Opt. Photonics, 12(2), 485-555, (2020).

8. Y. Liu, D. Marpaung, A. Choudhary, J. Hotten, \& B. J. Eggleton, "Link performance optimization of chip-based Si3N4 microwave photonic filters", J. Lightw. Technol, 36(19), 4361-4370, (2018).

9. V. J. Urick, M. E. Godinez, P. S. Devgan, J. D. McKinney \& F. Bucholtz, "Analysis of an analog fiber-optic link employing a low-biased Mach-Zehnder modulator followed by an erbium-doped fiber amplifier J. Lightw. Technol, 27(12), 2013-2019, (2009).

10. V. J. Urick, K.J. Williams, and J. D. McKinney. Fundamentals of microwave photonics. (John Wiley \& Sons, 2015).

11. E. Desurvire, Erbium Doped Fiber Amplifiers, (John Wiley \& Sons, 1994).

12. M. J. Connelly. Semiconductor optical amplifiers. (Kluwer Academic Publishers, 2002).

13. D. Pérez, I. Gasulla, J. Capmany, and R. A. Soref, "Reconfigurable lattice mesh designs for programmable photonic processors," Opt. Express 24, 12093-12106 (2016)

14. D. Pérez and J. Capmany. "Scalable analysis for arbitrary photonic integrated waveguide meshes." Optica 6(1) 19-27 (2019).

15. INPHENIX Semiconductor Optical Amplifier Device (Booster Type IPSAD1505 (1550nm)) https://www.inphenix.com/pro/Semiconductor\%20Optical\%20Amplifier/IPSAD1505.pdf

16. D. Pérez, I. Gasulla, J. Capmany. "Field Programmable Photonic Gate Arrays." Opt. Express 26(21), 2726527278 (2018). 